\title{
Development of pma/pvac-tpai-bmii solid polymer electrolytes for application in dye sensitized solar cell
}

\author{
Amisha Azmar ${ }^{1}$ and Tan Winie ${ }^{1,2}$ \\ ${ }^{1}$ Faculty of Applied Sciences, Universiti Teknologi MARA, 40450 Shah Alam, Selangor, Malaysia \\ ${ }^{2}$ Institute of Science, Universiti Teknologi MARA, 40450 Shah Alam, Selangor, Malaysia
}

\begin{abstract}
Electrolyte film of poly(methyl acrylate) (PMA) and poly(vinyl acetate) (PVAc) with composition of 90:10 and 20 wt.\% of tetrapropyl ammonium iodide (TPAI) at different of 1-butyl-3methyl imidazoliumiodide (BMII) concentration were prepared by solution casting technique. Highest conductivities achieve at $5 \mathrm{wt} . \%$ of BMII is $1.2 \times 10^{-11} \mathrm{~S} \mathrm{~cm}^{-1}$. Effects of temperature of this sample on the dielectric properties was studied by impedance spectroscopy. The dielectric constant, $\varepsilon_{\mathrm{r}}$ and dielectric loss, $\varepsilon_{i}$ increased with increasing temperature. Charge carrier relaxation time was extracted from the electrical modulus spectra. It was found that the relaxation time decreased with temperature. The ac conductivity was observed to obey the Jonscher's Universal Power Law. The correlated barrier hopping model (CBH) was used to interpret the conduction mechanism of the present electrolyte system. Electrolyte films were sandwiched between titanium dioxide photoanode and platinum counter electrode for dye-sensitized solar cells (DSSCs) assembly. The solar cell with $5 \mathrm{wt} . \%$ showed highest efficiency of $4.62 \%$ with maximum short circuit current density $\left(J_{s c}\right)$ of $10.04 \mathrm{mAcm}^{-2}$, open circuit voltage $\left(V_{o c}\right) 0.70 \mathrm{~V}$ and fill factor, $f f$ of $66.04 \%$.
\end{abstract}

\section{Introduction}

Nonrenewable energy need to be replaced with renewable energy source due to increasing human consumption. One of renewable energy that has been attracted by scientist is dye sensitized solar cells (DSSC) due to the potential to be low cost, flexible and environmental friendly alternatives energy source (Su'ait et al., 2015). A typical DSSC consists of a photo-electrode containing a dye sensitized mesoporous $\mathrm{TiO}_{2}$ layer coated on an ITO glass and a liquid electrolyte containing an iodide/triiodide redox mediator and a Pt metal counter electrode.(Arof et al., 2014). However, they suffer from electrolyte evaporation and leakage, corrosion of electrode, as well as other stability issues.

Polymer electrolytes are promising candidates to substitute liquid electrolytes. Solid polymer electrolyte (SPE) is one of the polymer electrolyte that has been recognized as the most flexible, light weight and leak proof novel material for ionic conducting devices(Cznotka et al., 2016; Winie \& Arof, 2004). It is formed by dissolving an alkali metal salt in a polymer that possesses donor atom such as $\mathrm{N}, \mathrm{O}$ or $\mathrm{S}$. The interaction between these atoms and cation of the salt leads to salt dissociation and hence ionic conduction. PMA and PVAc are good candidates as both consists of oxygen atom in their structure. Nonetheless, SPE has insufficient ionic conductivity at ambient temperature which limits its practical application.
Numerous approaches have been exploited by researchers to enhance the ionic conductivity by adopting plasticizers (Winie \& Arof, 2006; Woo et al., 2013), fillers (Winie et al., 2014) and ionic liquids (ILs) (Yusuf et al., 2017).ILs have been attracted attention due to their high ionic conductivity, low vapor pressure and high thermal stability (Sim et al., 2016). BMII is selected in this work as it can provide more ions to enhance ionic conductivity of polymer electrolyte.

The aim of the present work is to enhance the efficiency of DSSC on PMA/PVAc-TPAI at different concentration of BMII. These electrolyte were discussed based mainly on the ionic conductivity of the electrolyte films, dielectric properties, and its conduction mechanism.

\section{Experimental}

\subsection{Electrolyte preparation and characterization}

PMA dissolved in toluene was purchased from SigmaAldrich. PMA is first purified with methanol and then followed by the precipitation before being is dried for 24 hours under fume hood. The molecular weight of PMA is $47,000 \mathrm{~g} \mathrm{~mol}^{-1}$ determined using gel permeation chromatography (GPC) (Waters GPC (Milford US)). Polyvinyl acetate (PVAc) with molecular weight of 140,000 $\mathrm{g} \mathrm{mol}^{-1}$, Tetrapropyl ammonium iodide (TPAI) with purity $\geq 98 \%$ and 1 - 
butyl-3-methyl imidazoliumiodide (BMII) with purity $\geq 99 \%$ were obtained from Sigma-Aldrich. The required amount of PMA and PVAc was first dissolved in acetonitrile for 24 hours at room temperature. After complete dissolution of PMA/PVAc in acetonitrile, the required amount of TPAI and BMII was added. The solution was mixed and stirred continuosly at room temperature until a homogenous solution is obtained. The solution was casted in Teflon petri dish and allowed to evaporate slowly at room temperature to form films. Films of PMA/PVAc-TPAI-BMII were kept in a dessicator for continuos drying. Impedance measurement of the films were carried out using HIOKI 3532-50 LCR Hi-tester at a frequency range from $50 \mathrm{~Hz}$ to $1 \mathrm{MHz}$. The film is sandwiched between two stainless steel electrodes with diameter $1.2 \mathrm{~cm}$ under spring pressure. The ionic conductivity, $\sigma$ is calculated using equation:

$$
\sigma=t / R_{b} A
$$

where $t$ is the thickness of the film and $A$ is the filmelectrode contact area. The bulk resistance, $R_{b}$ is determined from the complex impedance plot. The complex impedance, $Z^{*}$ is given as:

$$
Z^{*}=Z_{r}+j Z_{i}
$$

where $Z_{r}$ and $Z_{i}$ are the real and imaginary parts of impedance, respectively.

Using the impedance data, the complex dielectric, $\varepsilon^{*}$ and electrical modulus, $\mathrm{M}^{*}$ can be obtained from:

$$
\varepsilon^{*}=\varepsilon_{r}+j \varepsilon_{i}
$$

with

$$
\varepsilon_{r}(\omega)=Z_{i} / \omega C_{o}\left(Z_{r}^{2}+Z_{i}^{2}\right)
$$

$$
\varepsilon_{i}(\omega)=Z_{r} / \omega C_{o}\left(Z_{r}^{2}+Z_{i}^{2}\right)
$$

and

$$
M^{*}=M_{r}+j M_{i}
$$

with

$$
\begin{aligned}
& M_{r}(\omega)=\varepsilon_{r} / \omega C_{o}\left(\varepsilon_{r}^{2}+\varepsilon_{i}^{2}\right) \\
& M_{i}(\omega)=\varepsilon_{i} / \omega C_{o}\left(\varepsilon_{r}^{2}+\varepsilon_{i}^{2}\right)
\end{aligned}
$$

Here, $\varepsilon_{\mathrm{r}}$ is the dielectric constant, $\varepsilon_{\mathrm{i}}$ is the dielectric loss, $M_{r}$ is the real part of electric modulus and $M_{i}$ is the imaginary part of electric modulus. Vacuum capacitance, $C_{0}=\varepsilon_{0} \mathrm{~A} / \mathrm{d}$, where $\mathrm{d}$ is the thickness of the electrolyte, $\mathrm{A}$ is surface area contact, $\varepsilon_{0}$ is the vacuum space permitivity of $8.85 \times 10^{-14} \mathrm{Fcm}^{-1}$ and $\omega$ is the angular frequency, $2 \pi \mathrm{f}$.

\subsection{DSSC fabrication}

The solid polymer electrolyte based DSSC layout glass/FTO/TiO $/ 2$ Dye/electrolyte/Pt/FTO/glass. Polymer electrolyte was sandwiching between the ruthenium dye $\mathrm{N} 719$ adsorbed in $\mathrm{TiO}_{2}$ electrode and platinum $(\mathrm{Pt})$ coated FTO glass. The DSSCs were characterized under the illumination of $100 \mathrm{~m} . \mathrm{Wm}^{-2}$ Xenon light source (Oriel LCS 100) with a Metrohm Autolab potentiostat (PGSTAT128 N).

\section{Results and discussion}

\subsection{Room temperature conductivity}

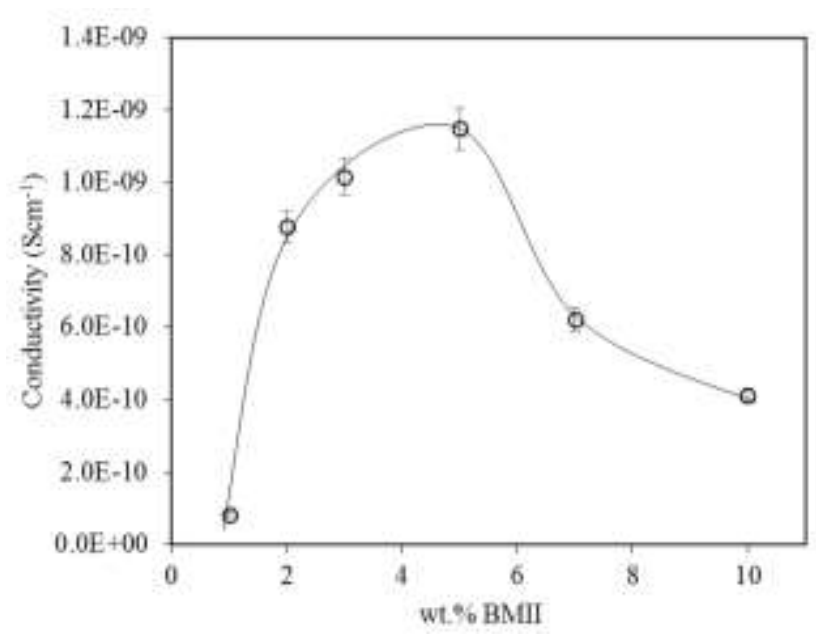

Fig. 1. Room temperature conductivity for PMA/PVAc-TPAI with respect to BMII concentration

The variation in conductivity with different BMII concentration for PMA/PVAc-TPAI is presented in Fig. 1. At 5 wt. $\%$ of BMII, the highest conductivity achieve at $1.15 \times 10^{-9} \mathrm{Scm}^{-1}$. From the impedance spectroscopy studies, the variation in conductivity as a function of BMII concentration could be understood on the basis of free ions concentration. The increase in conductivity is due to the fact that the addition of molten salt promotes more mobile ions into the polymer host. At 1-5 wt.\%, the conductivity increase as the rate of ion dissociation is higher than the rate of ion association. Conductivity start to decrease when BMII concentration increases to $7 \mathrm{wt} . \%$ as too many ions are provided in the polymer matrix. Hence, the distance between ion become too close and the rate of ions association is higher than rate of ion dissociation. The rate ions association such as ion pairs and ion aggregates do not take part in the conduction process leading to decline in the number of mobile ions which results to reducing the conductivity of the polymer system. Many previous researcher have already reported the similar trend (Ali, 2007; Winie, 2015; Woo et al., 2012). Moreover, the size of $\mathrm{BMI}^{+}$cation $(3.85 \AA)($ Liu et al., 2010) is smaller than TPAI (4.50 $\AA$ )(Gregor, 1968). Thus, $\mathrm{BMI}^{+}$cation can be mobile easier resulting increasing in conductivity.

\subsection{Temperature dependence conductivity}

Dielectric constant is a measure of the performance of a material to intensify electric flux and hence measures the ability of a given material to store electric charge(Tan et al.,2018). The charge exists from the dissociation of TPAI salt and BMII molten salt which 
produce a tetrapropyl ammonium, $\operatorname{Pr}_{4} \mathrm{~N}^{+}$and imidazolium, $\mathrm{BMI}^{+}$cation, respectively and Iodide, $\mathrm{I}^{-}$ anion. In the current study, the increasing number of charge increase a $\varepsilon_{\mathrm{r}}$ due to the enhancement in total polarization as temperature rose.(cf. Figure 2(a)) This phenomenon is attributed to the decrease in viscosity of the electrolyte, which increased the chain flexibility. The flexibility of polymer chains are greatly affected by motion of ions (Muhammad et al.,2017). Ions are more easily to move and aligned themselves in the direction of the applied electric field. Furthermore, increase in temperature also contributed to the increase in the number of free ions. Increase in temperature

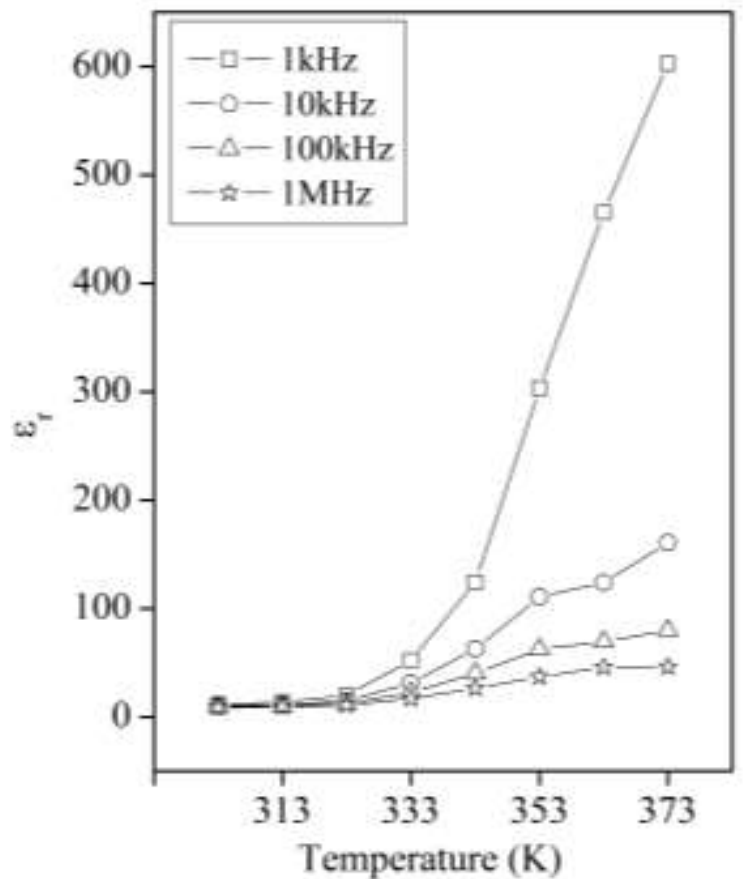

(a) increased the dissociation of salt into free ions. At low frequency, $(1 \mathrm{kHz})$, charge carriers get sufficient time to polarized before electric field was reversed. At higher frequencies, there was limited time for the charge to build up and caused $\varepsilon_{\mathrm{r}}$ value to decrease. Figure 2(b) depicts the variation of $\square_{i}$ as a function of temperature at selected frequencies. The effect of inertia which result from the changing direction of the electric field and alignment of ions at every cycle of applied electric field generated heat through internal friction and thus caused energy losses. The amount of energy losses is known as dielectric loss, $\varepsilon_{\mathrm{i}}$ (Tripathi et al.,2012).

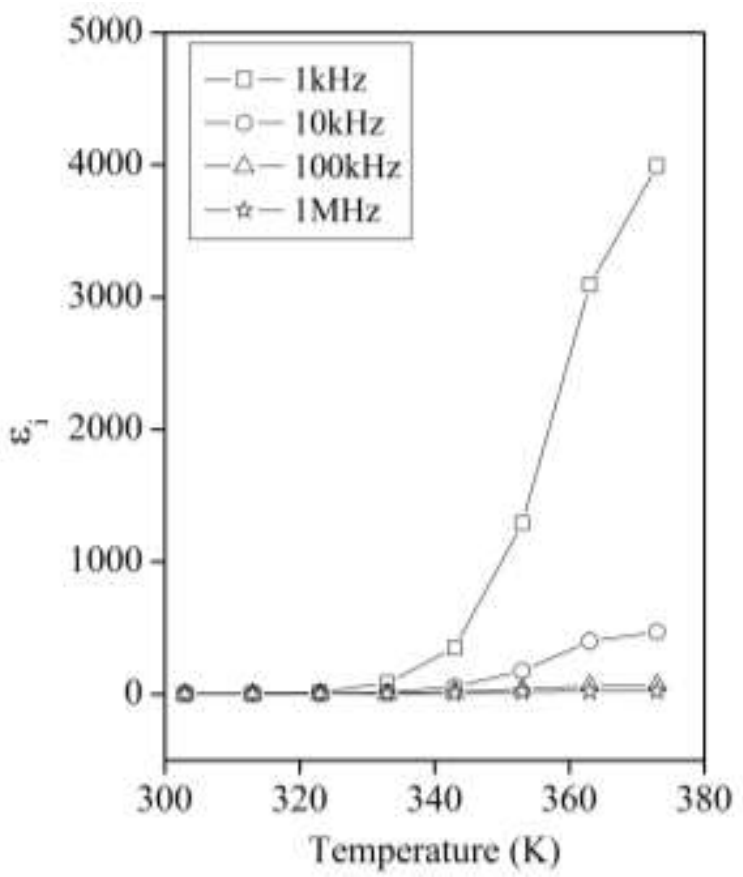

(b)

Fig. 2. (a) Dielectric constant, $\varepsilon_{\mathrm{r}}$ and (b) dielectric loss, $\varepsilon_{i}$ vs. temperature for 90/10(PMA/PVAc)-20wt.\% TPAI-5wt.\% BMII system at different frequency.

The effects of electrode polarization can be supressed using dielectric modulus analysis. Complex electric modulus has been used to study conductivity relaxation phenomena. Figure 3 (a) and (b) show the real part, $\mathrm{M}_{\mathrm{r}}$ and imaginary part, $\mathrm{M}_{\mathrm{i}}$ of electrical modulus as a function of frequency at various temperature for the same set of samples. It can be clearly observed that $\mathrm{M}_{\mathrm{r}}$ increased with temperatures in the high frequency region and approached zero with long tail at lower frequencies. The long tail indicates the suppression of electrode polarization(Woo et al., 2012).

Plotting the imaginary part of electrical modulus, $\mathrm{M}_{\mathrm{i}}$ with respect to frequency results in the manifestation of dispersion peaks as shown in figure 3(b). The occurrence of dispersion peaks suggest that the current electrolyte system are predominantly ionic conductors(Mellander \& Albinsson, 1996). In other words, the ionic motion and polymer segmental motion are strongly coupled. The capacitance values could be calculated at the peak frequency from the relation:

$$
M_{i}=\frac{\varepsilon_{0}}{2 C}
$$

The obtained capacitance values were in the range $0.5-1 \mathrm{pF}$, indicating that the dispersion peak observed is attributed to the bulk effect of the materials. As temperature increased, the peak frequency is observed to shift towards higher values. This indicates that conduction in the PMA/PVAc takes place through charge migration of ions between interaction sites of the polymer, along with its segmental relaxation, attesting that ionic conduction is predominant in the studied structures. The mobility of ionic charges carriers increased and causes the drop in relaxation time. The angular frequency of the applied field, $\omega$ at which the $M_{i}$ maxima occurs, defines the relaxation time for the ionic charge carriers, $\tau$ by the relation,

$$
\tau \omega \approx 1
$$

The occurrence of relaxation time is the result of the efforts carried out by the ionic carriers to obey the change in the direction of applied field (Ramly, 2011). 
Table 1 presents the values of conductivity and relaxation time for 90/10(PMA/PVAc)-20wt.\% TPAI5wt.\% BMII system at selected temperatures. As the temperature increased, ions experience an increase in mobility. The ion will be able to align themselves with the applied field in a shorter time when the field changes direction. Hence, relaxation time of ions decreased with temperature.

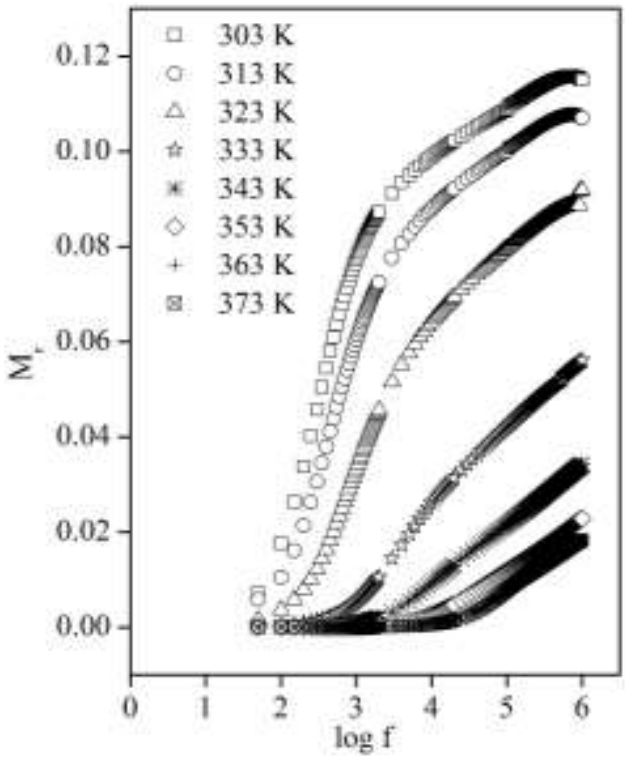

(a)

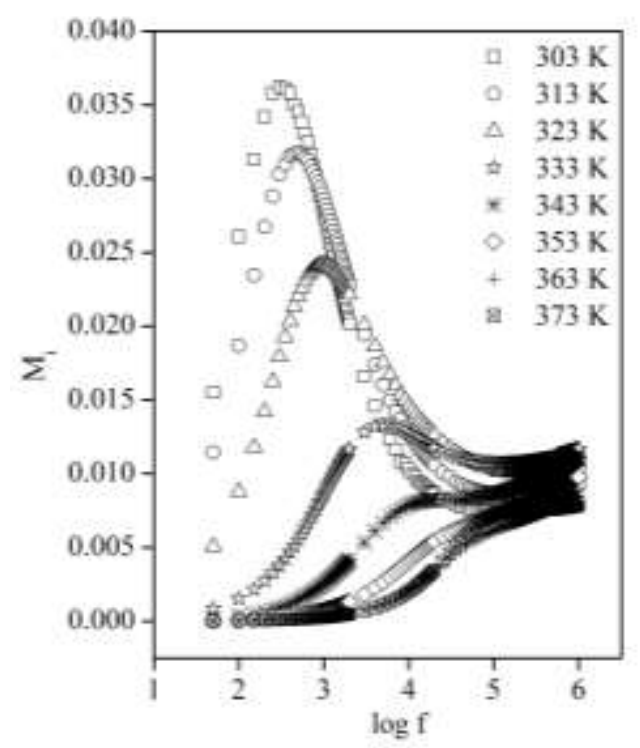

(b)

Fig. 3. Temperature dependent of (a) $M_{r}$ and (b) $M_{i}$ vs. log f for 90/10(PMA/PVAc)-20wt.\% TPAI-5wt.\% BMII system.

Table 1. The value of conductivity, $\sigma$ for 90/10(PMA/PVAc)-20wt.\% TPAI-5wt.\% BMII as a function of temperature.

\begin{tabular}{|c|c|c|}
\hline Temperature (K) & Conductivity (S/cm) & $\tau(\mathrm{s})$ \\
\hline 303 & $1.2 \times 10^{-9}$ & $5.3 \times 10^{-4}$ \\
\hline 313 & $1.6 \times 10^{-9}$ & $3.2 \times 10^{-4}$ \\
\hline 323 & $2.5 \times 10^{-8}$ & $1.7 \times 10^{-4}$ \\
\hline 333 & $1.7 \times 10^{-7}$ & $3.2 \times 10^{-5}$ \\
\hline 343 & $8.3 \times 10^{-7}$ & $8.0 \times 10^{-6}$ \\
\hline 353 & $3.3 \times 10^{-6}$ & $2.7 \times 10^{-6}$ \\
\hline 363 & $1.4 \times 10^{-5}$ & $1.8 \times 10^{-6}$ \\
\hline 373 & $4.5 \times 10^{-5}$ & $7.4 \times 10^{-7}$ \\
\hline
\end{tabular}

Ionic conduction mechanism of an electrolyte can be determined by employing Jonscher's universal power (Jonscher, 1996) as given by:

$$
\begin{gathered}
\sigma(\omega)=\sigma_{a c}+\sigma_{d c} \\
\sigma(\omega)=A \omega^{s}+\sigma_{d c}
\end{gathered}
$$

where $\sigma(\omega)$ is the total conductivity, $\sigma_{\mathrm{ac}}$ is the ac conductivity and $\sigma_{\mathrm{dc}}$ is the dc conductivity. The ac conductivity is represented by $\mathrm{A} \omega^{\mathrm{s}}$ where $\mathrm{A}$ is a temperature dependent parameter and $\mathrm{s}$ is power law exponent. The ac conductivity can also be obtained using:

$$
\sigma_{a c}=\varepsilon_{o} \varepsilon_{r} \omega
$$

By substituting $\sigma_{\mathrm{ac}} \mathrm{A} \omega^{\mathrm{s}}$ into equation 5, the value of exponent $\mathrm{s}$ was determined by plotting the following relation:

$$
\ln \varepsilon_{i}=\ln \frac{B}{\varepsilon_{O}}+(s-1) \ln \omega
$$

The frequency dependence of conductivity at different temperatures for 90/10(PMA/PVAc)-20wt.\% TPAI5wt.\% BMII system is shown in Figure 4. 


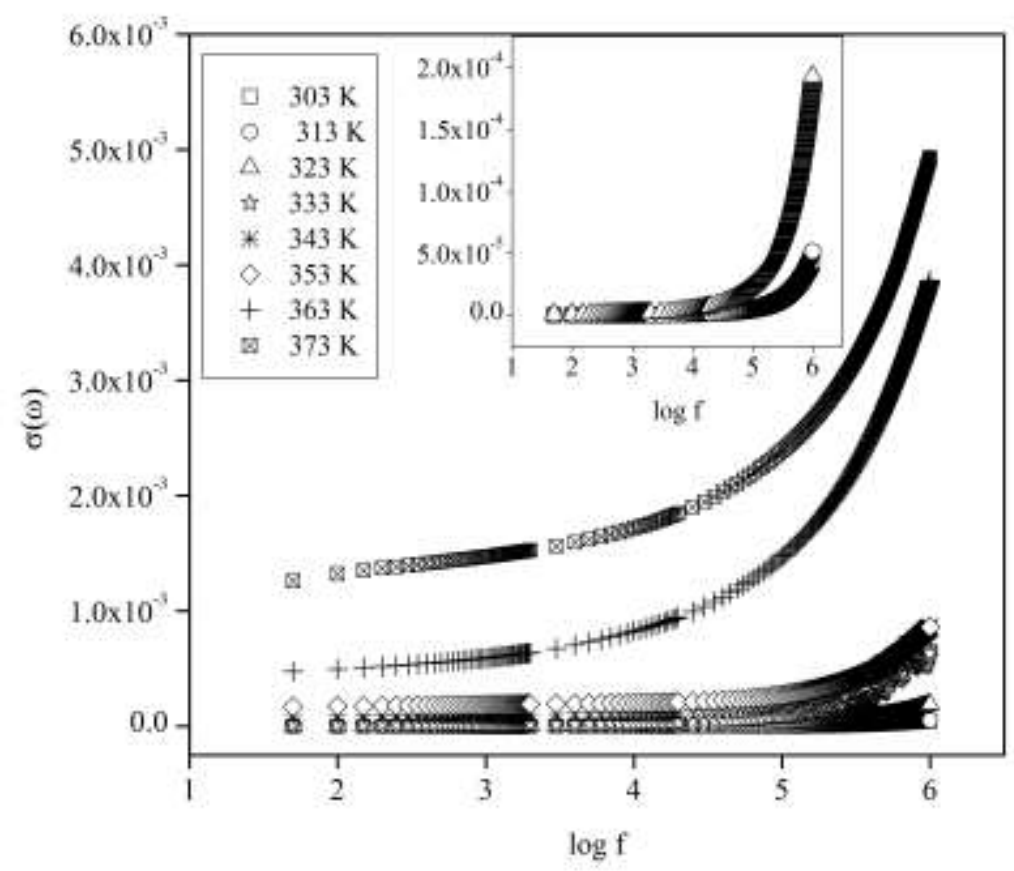

Fig. 4. Variation of AC conductivity with frequency for 90/10(PMA/PVAc)-20wt.\% TPAI-5wt.\% BMII system at different temperature.

The conductivity is found to be almost frequency independent at low frequency region and became frequency dependent at high frequency region. The trend of frequency dependence of conductivity observed in figure 4 is in agreement with the prediction of the jump relaxation model (Funke, 1997). Funke and Wilmer (2011) stated that ion can easily jump to its neighbouring vacant site at the lower frequency as it has enough time. The successful jump results in a long range translational motion of ions. After ion has arrived at the new complexation site, the ion either jumps back to its originals site (backward jump) or relax at the new site before next forward jump. Probability of both backward jump and relaxation increases with frequency. This leads to the presented conductivity dispersion at the high frequencies.

According to the Jump relaxation model, the power law exponent $\mathrm{s}$ from equation 14 is related to the rate of back jump to the site relaxation time as

$$
\mathrm{s}=\text { back jump rate/ site relaxation time }
$$

When $\mathrm{s}<1$, probability for the ion to relax at the new site is higher than the probability of the ion to jump back to its initial site.

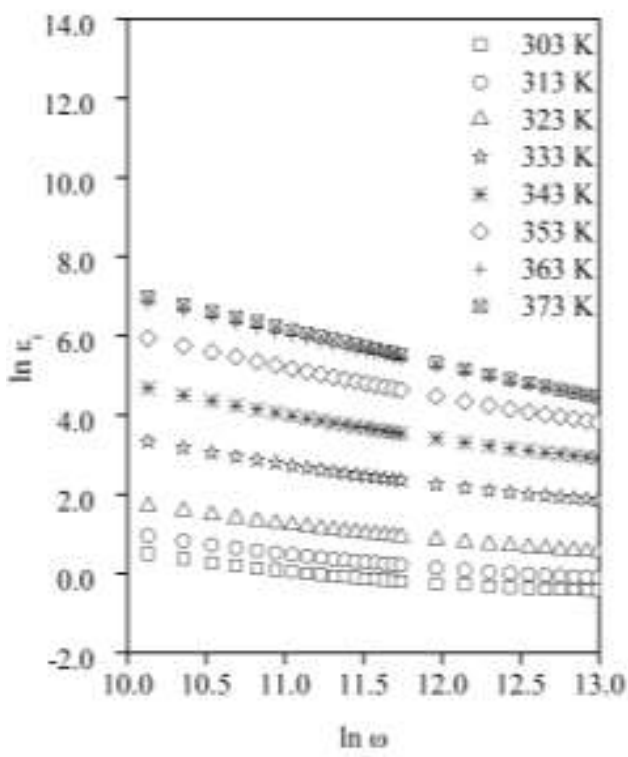

Fig. 5. $\ln \varepsilon_{i}$ vs. $\ln \omega$ at different temperatures for 90/10(PMA/PVAc)-20wt.\% TPAI-5wt.\% BMII. 


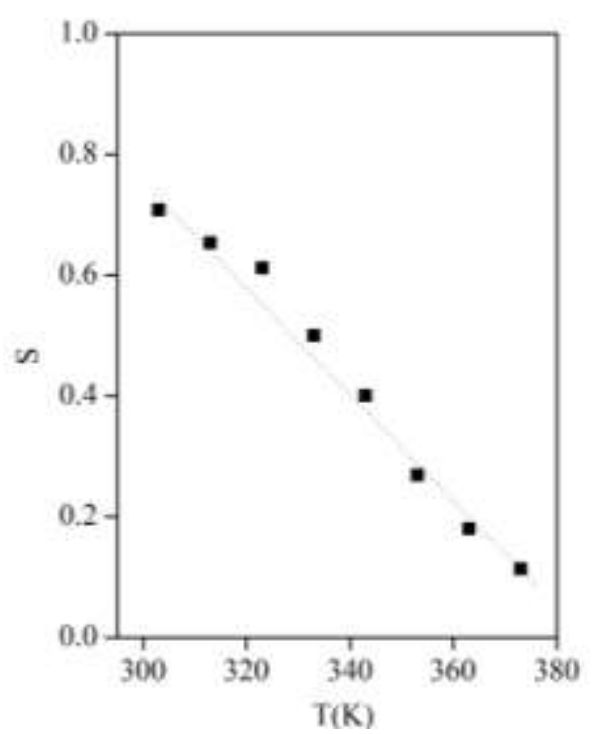

Fig. 6. Variation of the exponent s with temperature.

The value of s can be determined from the slope of $\ln \varepsilon_{\mathrm{i}}$ versus $\ln \omega$ as shown in figure 5. The variation of exponent $\mathrm{s}$ with temperature is plotted as shown in figure 6 and its value is in the range of 0.71 to 0.11 . The graph shows that $\mathrm{s}$ decreased with increasing temperature with a gradient 0.0019. Many different models have been proposed to describe the conduction mechanism of the materials with temperature dependence under the applied ac field. Two distinct relaxation processes, namely quantum mechanical tunnelling and classical hopping over barrier or some combinations between them, may be used to describe the ac conduction(Mansour et al.,2010). For example are quantum mechanical tunnelling (QMT) model (Muhammad, 2016), non-overlapping small polarons (NSPT) model (Kharrat et al. 2017), overlapping-largepolaron tunnelling (OLPT) model (Jamil et al. 2017), and many more. The obtained results imply that the correlated barrier hopping model $(\mathrm{CBH})$ is the most suitable model to describe the AC electrical conduction for 90/10(PMA/PVAc)-20wt.\% TPAI-5wt.\% BMII system. In this model, the carrier motion occurs by means of hopping over the Coulomb barrier separating two defect centres. This model, first developed by Pike (1972), for single-electron hopping, has been extended by Elliott (1987), for two electrons hopping simultaneously. The charge carrier is assumed to hop between site pairs over the potential barrier separating them (El-Nahass et al., 2014).

\subsection{DSSC performance}

Electrolytes of PMA/PVAc-TPAI containing different wt.\% BMII were assembled into DSSCs. Figure 7 and Table 2 show the $J-V$ curves and performance parameters of the assembled DSSCs. Solar cell parameters, under the irradiation of $1000 \mathrm{Wm}^{-2}$ such as open circuit voltage $\left(V_{o c}\right)$, and short current density $\left(J_{s c}\right)$ were measured and the fill factor $(f f)$ and efficiency $(\eta)$ were calculated using following formula,

$$
\begin{gathered}
f f=\frac{J_{\max } V_{\max }}{J_{s c} V_{o c}} \\
\eta=\frac{J_{s c} V_{o c} f f}{\text { total incident power density }}
\end{gathered}
$$

where $J_{\max }$ and $V_{\max }$ are the current density and voltage at maximum power output.

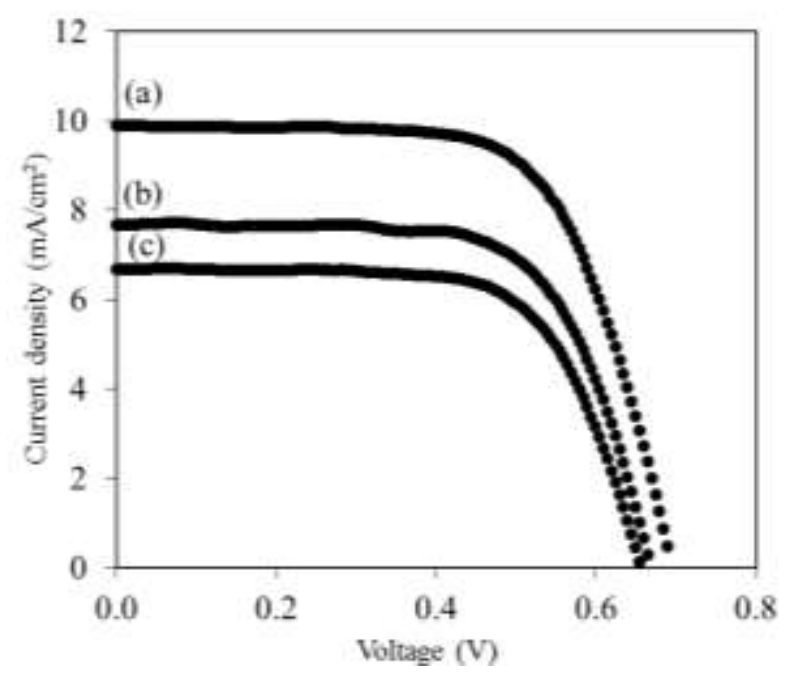

Fig. $7 \mathrm{~J}-V$ characteristics of DSSC assembled with PMA/PVAc-TPAI at (a) 5wt.\%, (b) 7wt.\% and (c) $3 w t . \%$ of BMII

Table 2. performance parameter for DSSC assembled of PMA/PVAc-TPAI at different BMII concentration

\begin{tabular}{|c|c|c|c|c|}
\hline wt.\% BMII & $V_{o c}(\mathrm{~V})$ & $J_{s c}\left(\mathrm{mAcm}^{-2}\right)$ & $f f(\%)$ & $\eta(\%)$ \\
\hline 3 & 0.66 & 6.83 & 66.89 & 3.02 \\
\hline 5 & 0.70 & 10.04 & 66.08 & 4.62 \\
\hline 7 & 0.67 & 7.84 & 67.08 & 3.52 \\
\hline
\end{tabular}


DSSC with 5 wt.\% of BMII shows higher efficiency of $4.62 \%$ compared to other composition with higher values of $J_{s c}$ of $10.04 \mathrm{mAcm}-2, V_{o c}$ of 0.70 $\mathrm{V}$ and $f f$ of $66.08 \%$.

Iodide conductivity is reflected in the value of $J_{s c}$ (Hassan et al. 2014; Mathew et al., 2013). Thus, higher value of $\mathrm{J}_{\mathrm{sc}}$ at $5 \mathrm{wt} \%$ of BMII can be attributed to higher iodide conductivity of the electrolyte due to higher number and mobility of free $\mathrm{I}^{-}$anions. $\mathrm{BMI}^{+}$is smaller cation than $\mathrm{Pr}_{4} \mathrm{~N}^{+}$as mention in section 1.1 before. Smaller cation can intercalate into the lattice of nano $\mathrm{TiO}_{2}$ causing a positive shift of the conduction band edge potential. The increase of driving force for the charge injection lead to higher $J_{s c}$ and efficiency (Dissanayake et al., 2012). The subsequent drop in $J_{s c}$ beyond 5 wt. $\%$ is mostly due to reduction of the number of free $I^{-}$ion as a result of ion association leading to the formation of ion pairs, triplets and ion aggregates which not participate in contribution of $J_{s c}$.

\section{Conclusion}

In this study, it is found that the ionic conductivity of these electrolytes has correlation with the dielectric strength and the polymer chain segmental motion relaxation time. The dielectric behavior proved that the values of $\varepsilon_{\mathrm{r}}$ and $\varepsilon_{\mathrm{i}}$ are strongly dependent on the frequency and temperature. The relaxation time extracted from the peak $M_{i}$ versus $\log$ frequency is found to decrease with conductivity and temperature. This study also confirms that the ions transportation for PMA/PVAc-TPAI-BMII described by the correlated barrier hopping model $(\mathrm{CBH})$. Increment in conductivity affects the performance of DSSC whereas 5 wt.\% of BMII shows highest value of conductivity and efficiency in the electrolyte system.

The authors would like to thank Faculty of Applied Sciences, Universiti Teknologi MARA for the support.

\section{References}

1. Ali, A.M.M.,Yahya., M.Z.A., Bahron, H., Subban, R.H.Y., Harun, M.K., Atan, I., 2007. Impedance Studies on Plasticized PMMA-LiX [X: $\left.\mathrm{CF}_{3} \mathrm{SO}_{3}{ }^{-}, \mathrm{N}\left(\mathrm{CF}_{3} \mathrm{SO}_{2}\right)_{2}{ }^{-}\right]$Polymer Electrolytes. Materials Letters, Volume 61(10), pp.2026-2029

2. Arof, A.K., Aziz, M.F., Noor, M.M., Careem, M.A., Bandara, L.R.A.K., Thotawatthage, C.A., Dissanayake, M.A.K.L., 2014. Efficiency Enhancement by Mixed Cation Effect in DyeSensitized Solar Cells with a PvDF based Gel Polymer Electrolyte. International Journal of Hydrogen Energy, Volume 39(6), pp. 2929-2935

3. Cznotka, E., Jeschke, S., Grünebaum, M., \& Wiemhöfer, H.-D., 2016. Highly-Fluorous Pyrazolide-based Lithium Salt in PVDF-HFP as Solid Polymer Electrolyte. Solid State Ionics, Volume 292, pp.45-51

4. Dissanayake, M.A.K.L., Thotawatthage, C.A., Senadeera, G.K.R., Bandara, T.M.W.J., Jayasundera, W. J.M.J.S.R., Mellander, B.E.,
2012. Efficiency Enhancement by Mixed Cation Effect in Dye-Sensitized Solar Cells with PAN based Gel Polymer Electrolyte. Journal of Photochemistry and Photobiology A: Chemistry, Volume 246, pp.29-35

5. El-Nahass, M.M., Atta, A.A., El-Zaidia, E.F.M., Farag, A.A.M., Ammar, A.H., 2014. Electrical Conductivity and Dielectric Measurements of CoMTPP. Materials Chemistry and Physics, Volume 143(2), pp.490-494.

6. Elliott, S.R., 1987. A.C. Conduction In Amorphous Chalcogenide and Pnictide Semiconductors. Advances in Physics, Volume 36, pp. 135-217

7. Funke, K.,1997. Ion Transport in Fast Ion Conductors - Spectra And Models. Solid State Ionics, Volume 94(1), pp. 27-33

8. Funke, K., Wilmer, D., 2011. Concept of Mismatch and Relaxation Derived from Conductivity Spectra of Solid Electrolytes. MRS Proceedings, Volume 548, pp. 403-414

9. Gregor, H.P., 1968. Ion-Exchange Membranes Correlation Between Structure and Function Pure and Applied Chemistry, Volume 16, pp. 329-350

10. Hassan, H.C., Abidin, Z.H.Z., Careem, M.A., Arof, A.K., 2014. Chlorophyll as Sensitizer In $\mathrm{I}^{-} / \mathrm{I}_{3}^{-}$-Based Solar Cells with Quasi-Solid-State Electrolytes. High Performance Polymers, Volume 26(6), pp.647-652

11. Jamil, A., Afsar, M.F., Sher, F., Rafiq, M.A., 2017. Temperature and Composition Dependent Density of States Extracted Using Overlapping Large Polaron Tunnelling Model in $\mathrm{MnxCo} 1-\mathrm{xFe}_{2} \mathrm{O}_{4} \quad(\mathrm{x}=0.25, \quad 0.5, \quad 0.75)$ nanoparticles. Physica B: Condensed Matter, Volume 509, pp.76-83

12. Jonscher, A.K., 1996. Universal Relaxation Law. Chelsea Dielectrics Press, London.

13. Kharrat, A.B.J., Moussa, S., Moutiaa, N., Khirouni, K., Boujelben, W., 2017. Structural, Electrical and Dielectric Properties of Bi-doped $\mathrm{Pr}_{0.8-\mathrm{x}} \mathrm{Bi}_{\mathrm{x}} \mathrm{Sr}_{0.2} \mathrm{MnO}_{3}$ Manganite Oxides Prepared by Sol-Gel Process. Journal of Alloys and Compounds, Volume 724, pp.389-399.

14. Liu, S., Liu, W., Liu, Y., Lin, J.-H., Zhou, X., J Janik, M., Zhang, Q., 2010. Influence Of Imidazolium-Based Ionic Liquids on the Performance of Ionic Polymer Conductor Network Composite Actuators, Polymer International, Volume 59(3), pp.321-328

15. Mansour, S.A., Yahia, I.S., \& Yakuphanoglu, F., 2010. The Electrical Conductivity and Dielectric Properties of C.I. Basic Violet 10. Dyes and Pigments, Volume 87(2), pp.144-148

16. Mathew, A., Anand, V., Rao, G.M., Munichandraiah, N., 2013. Effect of Iodine Concentration on the Photovoltaic Properties of Dye Sensitized Solar Cells for Various $\mathrm{I}_{2} / \mathrm{LiI}$ Ratios. Electrochimica Acta, Volume 87, pp. 9296.

17. Mellander, Albinsson, B.E., 1996. Electric and Dielectric Properties of Polymer Electrolytes. in: 
Chowdari, B.V.R, Dissanayake, M.A.K.L., and Careem, M.A. (Eds.), World Scientific, Singapore, pp. 83-96

18. Muhammad, F.H., Jamal, A.,Winie, T., 2016. Dielectric and AC Conductivity Behavior of Hexanoyl Chitosan-NaI Based Polymer Electrolytes. International Journal of Advanced and Applied Sciences, Volume 3(10), pp. 9-13

19. Muhammad, F.H., Jamal, A., Winie, T. 2017. Study on Factors Governing the Conductivity Performance of Acylated Chitosan-NaI Electrolyte System. Ionics. Volume 23(11), pp. 112

20. Pike, G.E., 1972. AC Conductivity of Scandium Oxide and a New Hopping Model for Conductivity. Physical Review B, Volume 6(4), pp. $1572-1580$

21. Ramly, K., Isa, M.I.N., Khiar, A.S.A., 2011. Conductivity and Dielectric Behaviour Studies of starch/PEO + x wt-\% $\mathrm{NH}_{4} \mathrm{NO}_{3} \quad$ Polymer Electrolyte. Materials Research Innovations, Volume 15, pp.82-85

22. Sim, L.N., Yahya, R., Arof, A.K. 2016. Blend Polymer Electrolyte Films based on Poly(Ethyl Methacrylate)/Poly(Vinylidenefluoride-CoHexafluoropropylene) Incorporated with 1-Butyl3-Methyl Imidazolium Iodide Ionic Liquid. Solid State Ionics, Volume 291, pp. 26-32

23. Su'ait, M.S., Rahman, M.Y.A., Ahmad, A. 2015. Review On Polymer Electrolyte In DyeSensitized Solar Cells (DSSCs). Solar Energy, Volume 115, pp.452-470

24. Tan, C.Y., Farhana, N.K., Saidi, N.M., Ramesh, S., Ramesh, K. 2018. Conductivity, Dielectric Studies and Structural Properties of P(VA-Co-PE) and Its Application In Dye Sensitized Solar Cell. Organic Electronics, Volume 56, pp.116-124.

25. Tripathi, S.K., Gupta, A., Kumari, M. 2012. Studies on electrical conductivity and dielectric behaviour of PVdF-HFP-PMMA-NaI polymer blend electrolyte. Bulletin of Materials Science, Volume 35(6), pp. 969-975

26. Winie, T., Arof, A.K. 2004. Dielectric Behaviour and $\mathrm{AC}$ Conductivity of $\mathrm{LiCF}_{3} \mathrm{SO}_{3}$ Doped $\mathrm{H}-$ Chitosan Polymer Films. Ionics, Volume10(3), pp. 193-199

27. Winie, T., Shahril., N.S.M., 2015. Conductivity Enhancement by Controlled Percolation of Inorganic Salt in Multiphase Hexanoyl Chitosan/Polystyrene Polymer Blends. Frontiers of Materials Science Volume, 9(2), pp. 132-140

28. Winie, T., Arof, A.K., 2006. Effect of Various Plasticizers on the Transport Properties of Hexanoyl Chitosan-based Polymer Electrolyte. Journal of Applied Polymer Science, Volume 101(6), pp. 4474-4479

29. Winie, T., Hanif, N.S.M., Chan, C.H., Arof, A.K., 2014. Effect of the Surface Treatment of the $\mathrm{TiO}_{2}$ Fillers on the Properties of Hexanoyl Chitosan/Polystyrene Blend-based Composite Polymer Electrolytes. Ionics, Volume 20(3), pp. 347-352

30. Woo, H.J., Majid, S.R., Arof, A.K., 2012. Dielectric Properties and Morphology of Polymer Electrolyte based on Poly(E-Caprolactone) and Ammonium Thiocyanate. Materials Chemistry and Physics, Volume 134(2-3), pp. 755-761

31. Woo, H.J., Majid, S.R., Arof, A.K., 2013. Effect of Ethylene Carbonate on Proton Conducting Polymer Electrolyte based on Poly(ECaprolactone) (PCL). Solid State Ionics, Volume 252, pp. 102-108.

32. Yusuf, S.N.F., Yahya, R., Arof, A.K., 2017. Ionic Liquid Enhancement of Polymer Electrolyte Conductivity and their Effects on the Performance of Electrochemical Devices. in: Handy, S., (Eds.), Progress and Developments in Ionic Liquids, London pp. 157-183 